\title{
Des feux dans la vallée
}

\section{Emmanuel Ghesquière}

\section{OpenEdition}

\section{Journals}

Édition électronique

URL : http://journals.openedition.org/rao/941

DOI : $10.4000 /$ rao 941

ISBN : 978-2-7535-1609-0

ISSN : 1775-3732

Éditeur

Presses universitaires de Rennes

Édition imprimée

Date de publication : 31 décembre 2009

Pagination : 312-313

ISBN : 978-2-7535-1086-9

ISSN : 0767-709X

\section{Référence électronique}

Emmanuel Ghesquière, "Des feux dans la vallée », Revue archéologique de l'Ouest [En ligne], 26 | 2009,

mis en ligne le 31 décembre 2011, consulté le 04 décembre 2020. URL : http://

journals.openedition.org/rao/941 ; DOI : https://doi.org/10.4000/rao.941 
Marchand, G. (dir.), 2009 - Des feux dans la vallée. Les habitats du Mésolithique et du Néolithique récent de l'Essart à Poitiers, Rennes, PUR, coll. "Archéologie \& Culture », 246 p. (ISBN : 978-2-7535-0834-7; 24€).

Cette publication monographique présente de façon complète les résultats de l'investigation partielle d'un dôme formant une " île » dans le lit majeur du Clain, immédiatement au nord de Poitiers. Les principales caractéristiques du site concernent un secteur décapé sur une surface totale de $2000 \mathrm{~m}^{2}$. Trente-neuf foyers aménagés, certains superposés, rythment la surface du décapage. De nombreuses zones de dispersion de pierres calcaires brûlées parsèment la surface et correspondent à des déstructurations de foyers. Le mobilier découvert en association avec ces foyers et, plus généralement, dans la couche archéologique, concernent des vestiges du Mésolithique moyen, du Mésolithique récent/ final et du Néolithique récent. La compression des niveaux n’a pas permis de séparer strictement les différentes couches d'occupations mésolithiques.

Le principal apport de la fouille est cette extraordinaire concentration de foyers en bordure de rivière, qui laisse présumer une récurrence des occupations sur cette île, avec ses implications sociales pour l'acquisition et la transformation possible de denrées alimentaires; la pêche et le traitement de produits halieutiques sont ici justement proposés.

Le volume s'articule en plusieurs chapitres d'inégale longueur. Le premier, introductif, par Grégor Marchand, retrace de façon intelligente et approfondie la méthodologie adoptée sur la fouille et les premières observations et interrogations sur la période mésolithique.

Vient après un second chapitre qui traite des données géomorphologique mises en évidence sur le site (Farid Sellami). Ces données remettent en situation la dynamique sédimentaire complexe de l'île, qui a permis, par des dépôts alluvionnaires successifs, la préservation des niveaux anciens.

L'ensemble des structures archéologiques, largement dominées par celles liées à la combustion, constitue le troisième chapitre. L'étude précise de certains foyers permet de mettre en évidence les phénomènes d'exposition à la chaleur et d'érosion des blocs de calcaire formant l'agencement des foyers. Les phénomènes de déstructuration et de réalisation de ces agencements permettent d'entrevoir une occupation encore plus complexe et plus dense que celle révélée par les déjà nombreux foyers en place.

L'ensemble des principaux chapitres qui suivent constitue une part importante de la publication. Traités par Grégor Marchand et Sylvène Michel, ils reprennent l'ensemble des apports de la production lithique à la compréhension du site. La première partie concerne l'étude des techniques de débitage, qui mettent en évidence plusieurs méthodes distinctes. La première, majoritaire, se rapporte globalement au style de Montbani et à des dérivés. Ils correspondent à une production de supports lamino-lamellaires par la méthode de la percussion indirecte. La seconde correspond en revanche à un style moins régulier dont une partie des nucléus et des supports produits pourraient relever de l'occupation la plus ancienne du site (Mésolithique moyen).

L'étude des armatures a été réalisée à partir d'une description typologique très précise qui va permettre plusieurs divisions strictes. Les armatures caractéristiques du second Mésolithique vont ainsi être individualisées; elles rassemblent la majorité du corpus. Elles correspondent dans l'ensemble à des formes triangulaires ou trapézoïdales, dont certains exemplaires présentent une retouche inverse rasante. Parmi les plus emblématiques, on peut citer de nombreux trapèzes de Téviec, des armatures de Sonchamp, des trapèzes du Payré et des flèches de Montclus, qui témoignent de multiples influences stylistiques. La seconde série d'armatures est dominée par les triangles scalènes, parfois de Montclus, complétée par quelques triangles isocèles et pointes à base retouchée. Si la compression des niveaux n'a pas permis de reconstituer les corpus par ensemble chronoculturel, en revanche, la cotation altimétrique de toutes les armatures a permis de pallier certaines carences du gisement. Ainsi, les moyennes de profondeur de certaines des armatures a permis de séparer l'ensemble des artefacts du Mésolithique moyen de ceux du Mésolithique final mais également, au sein du second Mésolithique, de distinguer les trapèzes du Payré et les flèches de Montclus. Cette prouesse, qui n'avait rien d'évident sur une telle stratigraphie compressée, nous montre tout l'intérêt de l'emploi d'une méthode de relevé lourde. La succession de ces deux types d'armatures, leur origine typologique méditerranéenne et leur importance dans le processus de néolithisation des derniers chasseurs-cueilleurs sont en effet au cœur du vaste débat des débuts de l'agriculture dans l'ouest de la France.

Le reste de l'outillage est ensuite décrit. Largement dominé par les éclats diversement retouchés (815 pièces), il comprend également une série importante de lames à retouche Montbani. Les grattoirs forment eux aussi une série conséquente mais ne représentent même pas une pièce par foyer. En dehors des lames Montbani, les outils communs, impossible à trier entre ceux appartenant au Mésolithique moyen, final et au Néolithique récent, offrent peu d'intérêt d'étude suivant les auteurs. Le champ typologique extrêmement limité toutes périodes confondues reflète cependant une réduction drastique des usages qu'il est intéressant de relier au contexte socio-économique particulier à ce gisement (occupation temporaire spécialisée?). 
Après une étude de la céramique attestant de son attribution chronoculturelle au Néolithique final, la dernière partie, rédigée par Grégor Marchand et Sylvène Michel, replace les séries de l'Essart dans leur contexte chronoculturel global du premier et du deuxième Mésolithique. Ils réalisent là une synthèse approfondie qui complète les données déjà publiées par les deux auteurs.

La conclusion enfin reprend quelques aspects ethnographiques de la principale composante du site : les feux, en valorisant leur situation au bord du Clain.
D'un point de vue économique et social, les apports de la fouille et de la publication sont énormes et rappellent la complexité de ces ultimes chasseurs-cueilleurs. Grégor Marchand signe là un des ouvrages monographiques de référence sur la période pour le nord de la France avec, il ne faut pas l'oublier, une des plus grosses série d'armatures issue de fouilles.

Emmanuel GHESQUIÈre

(INRAP Basse-Normandie et UMR6566 - CreAAH)

TARrÊte, J. et Le Roux, C.-T. (coord.), 2008 - Le Néolithique, Paris, ministère de la Culture et de la Communication/ Picard, 424 p. (ISBN 978-2-7084-0801-2; $110 €$ ).

L’ouvrage, coordonné par Jacques Tarrête et CharlesTanguy Le Roux et qui s'ouvre sur une préface de Jean Guilaine, inaugure une nouvelle collection («Archéologie de la France »), coéditée par les éditions Picard et le Ministère de la Culture et de la Communication (Direction de l'Architecture et du Patrimoine) : Il s'agit d'un volumineux ouvrage grand format $(28 \times 24 \mathrm{~cm})$ de 415 pages (plus un index des sites archéologiques), qui fait le bilan des acquis récents de la recherche sur le Néolithique de la métropole et dans une moindre mesure de la Corse. Il réunit les articles d'une trentaine d'auteurs et d'une vingtaine de collaborateurs issus de l'ensemble du " paysage archéologique français » (CNRS surtout, suivi des agents de l'État, de l'université, des collectivités territoriales, puis de l'INRAP), qui explorent tour à tour les grands domaines d'étude de la Préhistoire récente : le rapport de l'homme à son environnement, l'habitat et l'organisation sociale, les vestiges mobiliers (fabrication, matériaux), les pratiques funéraires, les relations sociales et les manifestations artistiques. Les chercheurs mis à contribution sont parmi les meilleurs dans leurs spécialités et offrent des synthèses très richement illustrées (il s'agit ici d'un « beau livre »), ponctuées par des encarts consacrés aux méthodes mises à contribution pour la recherche en Préhistoire : datations radiocarbone, analyse chimique des matériaux, tracéologie, expérimentation, analyse $\mathrm{ADN}$, etc.

Les chapitres sont précédés d'une préface de Jean Guilaine. Véritable introduction à l'ouvrage, elle replace le Néolithique français dans le vaste mouvement qui couvre le Proche Orient et une bonne partie de l'Europe. L'auteur y évoque, dans de courts bilans historiographiques, les principales avancées dans chacune des thématiques qui forment les chapitres du livre. C'est aussi pour ce chercheur l'occasion de proposer de nouvelles pistes d'études et de partager avec le lecteur ses souhaits en matière d'évolution de la recherche.
La préface est suivie de six cartes permettant de saisir l'emprise des groupes néolithiques entre les $\mathrm{VI}^{e}$ et $\mathrm{III}^{\mathrm{e}}$ millénaires. Cet exercice cartographique, des plus périlleux lorsque l'on sait à quel point les néolithiciens peuvent se quereller à propos de la répartition des groupes culturels au cours du temps, n'échappera pas à la critique. Plutôt que de fustiger ce travail, il faut plutôt examiner ces cartes comme un état de la recherche et un bel effort de synthèse formant une bien utile introduction pour qui veut poursuivre plus avant la lecture de l'ouvrage. On regrettera toutefois ici l'absence d'un tableau chronologique reprenant ces données de manière synthétique. En effet, le seul cadre chronologique proposé avant la lecture du corps de l'ouvrage va du Tardiglaciaire à nos jours et a pour objet les évolutions environnementales.

Les 26 chapitres qui suivent sont regroupés en cinq grandes parties. Ils traitent de l'ensemble des données disponibles sur le Néolithique en laissant souvent une large place à l'interdisciplinarité, fait encore trop rarement mis en avant dans les synthèses parues ces dernières années qui font généralement la part belle à la seule culture matérielle. Certains pourront, bien entendu, pointer du doigt les lacunes ou les absences dans certains chapitres (en particulier la relative discrétion des données issues de l'archéologie préventive). D'autres pourront incriminer le choix des auteurs, certains étant absents du " casting " alors qu' ils font figure de références internationales; il reste que ce travail est monumental. C'est l'aboutissement d'un projet lancé il y a plus de 10 ans; malgré cette longue gésine qui pourrait laisser présager un ouvrage obsolète, il est important de souligner que la plupart des chapitres ont été réactualisés par leurs auteurs ces dernières années (les premières livraisons datent de 2000, beaucoup ont été réécrites en 2005 et 2006), permettant donc aux lecteurs de profiter des dernières avancées scientifiques dans certains domaines. 